Service social

\title{
Les représentations sociales de l'alimentation, de la santé et de la maladie des jeunes enfants
}

\section{Catherine Garnier, Lynn Marinacci et Martine Quesnel}

Volume 53, numéro 1, 2007

URI : https://id.erudit.org/iderudit/017991ar

DOI : https://doi.org/10.7202/017991ar

Aller au sommaire du numéro

Éditeur(s)

École de service social de l'Université Laval

ISSN

1708-1734 (numérique)

Découvrir la revue

Citer cet article

Garnier, C., Marinacci, L. \& Quesnel, M. (2007). Les représentations sociales de l'alimentation, de la santé et de la maladie des jeunes enfants. Service social, 53(1), 109-122. https://doi.org/10.7202/017991ar
Résumé de l'article

L'alimentation est devenue une préoccupation importante des sociétés occidentales en raison de ses conséquences sur la santé et le développement de l'enfant. Le présent article fera état, à partir du cadre théorique des représentations sociales, des résultats d'une classification descendante hiérarchique et d'une analyse factorielle du champ représentationnel lié à l'alimentation, la santé et la maladie des jeunes enfants ( 90 : maternelle et première année). On y retrouvera les principales dimensions qui organisent le champ de signification des représentations sociales et leur positionnement selon diverses variables socio-économiques. L'articulation équivoque des représentations sociales de l'alimentation, de la santé et de la maladie, en témoignant des interférences qui agissent dans l'environnement social des jeunes enfants, prêche en faveur des interventions en service social portant sur les aspects socioculturels de l'alimentation. 


\section{Les représentations sociales de l'alimentation, de la santé et de la maladie des jeunes enfants}

Catherine GARNIER

Professeure associée

Département de kinanthropologie

Faculté des sciences de l'éducation

Université du Québec à Montréal (UQAM)

Lynn MARINACCI

Agente de recherche et de planification

Groupe d'étude sur l'interdisciplinarité

et les représentations sociales (GEIRSO)

Université du Québec à Montréal (UQAM)

Martine QUESNEL

Doctorante

Programme des sciences humaines appliquées et agente de recherche au Groupe d'étude sur l'interdisciplinarité et les représentations sociales (GEIRSO) Université du Québec à Montréal (UQAM)

L'alimentation est devenue une préoccupation importante des sociétés occidentales en raison de ses conséquences sur la santé et le développement de l'enfant. Le présent article fera état, à partir du cadre théorique des représentations sociales, des résultats d'une classification descendante hiérarchique et d'une analyse factorielle du champ représentationnel lié à l'alimentation, la santé et la maladie des jeunes enfants ( 90 : maternelle et première année). On y retrouvera les principales dimensions qui organisent le champ de signification des représentations sociales et leur positionnement selon diverses variables socio-économiques. L'articulation équivoque des représentations sociales de l'alimentation, de la santé et de la maladie, en témoignant des interférences qui agissent dans l'environnement social des jeunes enfants, prêche en faveur des interventions en service social portant sur les aspects socioculturels de l'alimentation.

Mots clés : représentations sociales, alimentation, santé, maladie, enfants. 
Dysfunctional feeding has become an important preoccupation of occidental societies due to the consequences on the health and development of the child. The present article will present, within a theoretical context of social representations, results of a descending hierarchical classification and of a factorial analysis of a graphical field of nutrition linked to health and sickness of young children (90: kindergarten and first grade). We will find the principal dimensions that organize the signification of the field of social representation and their position according to the diverse socioeconomic variables. Equivocal articulation of social representation of alimentation, health and disease testifies of the interferences that play's a role in social environment of young children and that goes on in favour of social service intervention of alimentation sociocultural aspects.

\section{Key Words: social representations, feeding, health, sickness, children.}

\section{PROBLÉMATIQUE}

L'alimentation est une préoccupation importante des sociétés en raison de l'entrecroisement de ses dimensions politique, environnementale, biomédicale, économique et socioculturelle (Sobal et al., 1998). Dans les sociétés occidentales, la dimension biomédicale est bien documentée (Colver et al., 2005; Marshall et al., 2005; Morgan, 2005) en raison des problèmes de santé qui y sont liés (caries dentaires, allergies, anémie, ostéopénie, etc.). C'est l'obésité qui retient l'attention (Ells et al., 2005; Rennie et al., 2005), car l'obésité juvénile est aussi associée à une plus grande prévalence du diabète de type 2 (Mazza et al., 2005). L'obésité juvénile s'observerait autant au Canada, qu'en Australie, aux États-Unis, en France, en Grande-Bretagne et au Japon (MSSS, 2005). Au Québec, bien que ce problème touche $15 \%$ des jeunes, on constate que près du tiers des enfants de 9 ans s'efforcent de perdre du poids (Institut de la statistique du Québec, 1999). II se peut que ce contrôle résulte des pressions sociales qui sont exercées envers les personnes obèses. En effet, Lehman (2002) a observé la discrimination dont celles-ci sont l'objet dans les milieux médicaux et scolaires, en raison d'un préjugé sur l'absence de contrôle personnel sur des comportements à risque en matière d'alimentation (consommation élevée de sel, sucre et gras et faible consommation de fruits et de légumes: Koletzko et al., 2002). Concernant les jeunes enfants de l'ordre d'enseignement du primaire, Savoie-Zajc (2005) retient l'obésité comme un important facteur d'exclusion.

Eliadis (2006) souligne que l'obésité juvénile fait surtout l'objet d'interventions médicales ou diététiques, alors qu'elle nécessiterait des interventions en matière de travail social, parce qu'elle est liée à des changements sociaux, en particulier, des changements dans les styles de vie qui affectent la famille. L'approfondissement des savoirs relatifs au milieu d'appartenance génère un impact sur l'intervention sociale et sanitaire en ce qui concerne la dimension des croyances, des connaissances et des comportements (Jorm et al., 2005; Provencher, 2003). Sher et al., 2005, dans la discussion sur les facteurs d'adhésion à un traitement, invoquent la nécessité de former les intervenants sur l'impact des croyances déterminantes du milieu d'appartenance. La 
dimension socioculturelle de l'alimentation devrait être davantage étudiée, selon Lehman (2002), afin de susciter davantage d'interventions en matière de travail social.

Comme le constate Jacquinot (2002) concernant la nutrition des enfants, ceux-ci font face à des contradictions des discours familiaux, scolaires, télévisuels ou publicitaires, qui sont difficiles à concilier. Par ailleurs, Holub et al., 2005 mentionnent que les mots utilisés par les adultes dans ces discours sont souvent mal compris par les jeunes enfants. Dans cette même veine, Bruss et al., 2003 relèvent que les conflits entre les valeurs socioculturelles, les attentes familiales, les croyances et attitudes traditionnelles liées à l'alimentation, ainsi que les connaissances relatives à la nourriture et la maladie, constituent un thème central émergeant de leur analyse qui est lié à plusieurs facteurs socioculturels et familiaux concernant la prévention de l'obésité. La poursuite de leurs travaux leur a permis de comprendre comment les enfants de divers milieux ethniques composent avec des messages qui portent sur l'alimentation et l'obésité provenant de sources concurrentes (socioculturelles, familiales et du milieu de la nutrition) et qui recèlent divers niveaux de signification (Bruss et al., 2005). Les influences culturelles, que ces derniers auteurs rapprochent du concept d'archétypes, s'avèrent déterminantes pour comprendre les croyances, valeurs, attitudes et pratiques alimentaires des enfants. Par ailleurs, Lowes et Tiggemann (2003) font observer que sur les 135 enfants âgés de cinq à huit ans qui ont participé à leur étude, une proportion substantielle avait déjà internalisé les croyances sociétales concernant l'image corporelle idéale et particulièrement celle selon laquelle la diète constitue un des moyens privilégiés pour y parvenir.

D'un point de vue plus général, l'alimentation est considérée par Lévy-Strauss (1971) comme un langage traduisant la structure d'une société, et par Lahlou (1994) comme un vecteur de symbole et de croyance, en révélant des noyaux de sens collectifs. II s'agit d'un indice culturel qui sous-tend une organisation sociale et réfère à des rites et des rythmes de vie (Costalat-Founeau et al., 2002). Ivie (2005) précise que l'évaluation des croyances collectives permet de mieux diagnostiquer les sources de conflits potentiels qui agissent dans l'environnement social et qui interfèrent avec les pratiques d'intervention sociale. Bien que l'intériorisation opérée par les enfants des discours contradictoires des adultes à propos de l'alimentation, la santé et la maladie demeure peu documentée, il est possible de l'étudier à l'aide du cadre théorique des représentations sociales (Corsaro, 1990; Duveen, 1999). Ainsi, le présent article présentera les résultats d'une analyse des représentations sociales liées à l'alimentation, à la santé et à la maladie des jeunes enfants (maternelle et première année). Seront décrites les principales dimensions qui en organisent le champ de signification ainsi que leur positionnement sur ces dimensions selon le milieu d'appartenance et le niveau d'âge, et ce, afin de pouvoir guider les pratiques d'intervention sociale auprès des jeunes enfants et de leur milieu d'appartenance. 


\section{CADRE THÉORIQUE}

Les processus symboliques et les représentations sociales dans la représentation alimentaire ont été étudiés par Lahlou (1994) et par Masson et Moscovici (1997). Toutefois, l'ampleur du cadre théorique des représentations est à la source de diverses ambiguïtés. II existe plusieurs corpus théoriques qui traitent des représentations, dont deux courants principaux. L'un traite des représentations mentales dans lesquelles la représentation est un ensemble de connaissances ou de croyances, encodées en mémoire et que l'on peut extraire et manipuler mentalement (Dortier, 2003). Dans ce courant, plusieurs écoles de pensée s'affrontent sur les contenus des représentations, et la polémique persiste sur la suprématie du mot ou de l'image. Il existe également plusieurs écoles de pensée dans l'autre courant, celui sur les représentations sociales. Un consensus s'est toutefois établi sur leurs contenus, soit un savoir de «sens commun », de «systèmes de valeurs, de notions et de pratiques » (Moscovici, 1990, 1993) formés d'images, de symboles et de concepts, qui sont stables dans le temps et cohérents dans leurs contenus. À la différence des représentations mentales, le concept de représentations sociales ne peut se dissocier de la notion de groupe car une des caractéristiques essentielles des représentations sociales, c'est qu'elles sont partagées. C'est par l'objectivation qu'un groupe transforme l'abstrait en concret, produit des images qui favorisent la circulation du savoir de sens commun. Mais c'est par l'ancrage que de nouveaux savoirs sont incorporés dans le réseau de catégories qui existent déjà dans un groupe (Abric, 1994; Moscovici, 1990, 1993). Ces deux processus fondent d'ailleurs l'élaboration des représentations sociales. Markova (2000), paraphrasant Moscovici, précise que les représentations sociales sont caractérisées par des organisations relationnelles et dynamiques des savoirs et du langage de sens commun. Ce sont ces caractéristiques relationnelles et surtout dynamiques qui font que les représentations sociales, comme la culture, le langage ou la cognition, ne peuvent être appréhendées entièrement dans leur intégralité. Axé sur l'étude des phénomènes en perpétuels changements, le concept de changement social est central à la théorie des représentations sociales selon Markova (2000) et l'ensemble des travaux de Garnier (Garnier et al., 2004; Garnier et Doise, 2002; Garnier, 2002; Garnier et Rouquette, 2000; Rouquette et Garnier, 1999), dont, en particulier, ceux sur la pensée sociale (Garnier, 2002), qui l'attestent bien.

Les représentations sociales se manifestent par des organisations de contenus ancrées au centre de la vie sociale et de la réalité quotidienne des individus, groupes et sociétés, et qui sont essentiellement dynamiques, mais aussi symboliques (Markova, 2000). Cavallo et lannaccone (1993), se référant à Moscovici (1984), soulignent à propos des contenus symboliques qu'il s'agit d'extraire dans les analyses, les éléments figuratifs de la représentation qui se présentent «comme un système d'images rendant visible un grand nombre d'idées » (Cavallo et lannaccone, 1992, p. 4). Parmi le corpus de connaissances méthodologiques citées par ces derniers auteurs comme ayant permis de confirmer les fondements théoriques des représentations sociales, figurent un ensemble d'auteurs qui ont utilisé des épreuves d'associations de mots, dont les travaux les plus fondamentaux ont été réalisés par De Rosa (1988) et Abric (1994). 


\section{MÉTHODOLOGIE}

Comparant les résultats de huit années de recherche sur la maladie mentale, De Rosa (1988) note que le «choix des instruments d'enquête affectent de façon notoire le niveau de représentations étudiées » (De Rosa, 1988, p. 29). Les techniques verbales structurées (questionnaires avec échelles de distances sociales ou questions fermées ou questions ouvertes, entrevues dirigées ou semi-dirigées) permettent d'accéder à des éléments plus éloignés des contenus essentiels de la représentation sociale, alors que les associations libres, en raison de leur caractère projectif, permettent d'accéder à leur substrat symbolique ou leur essence figurative. Ceci constitue dans le cadre de l'intervention sociale un instrument utile pour comprendre les dynamiques de groupe aussi bien chez les adultes que chez les jeunes enfants. La méthode des associations libres à partir des mots inducteurs (Biber, 1996; Courtial, 1997; Clémence, 1995, 2001; Doise et al., 1992; Lacassagne et al., 2001) a été utilisée par l'équipe de recherche auprès des jeunes enfants en situation d'apprentissage de l'écriture, soit des élèves de maternelle et de première année du primaire. De courtes entrevues ont donc été réalisées afin de recueillir des induits, qui étaient des mots associés à trois groupes d'inducteurs liés à la nourriture (aliment et manger), la santé (santé) et la maladie (malade). Ces induits ont été recueillis auprès de 90 élèves de maternelle (40\%) et de première année (60\%) provenant de trois écoles. De ces 90 élèves, $46 \%$ étaient des filles et $54 \%$ étaient des garçons. Ces écoles étaient contrastées sur les plans socioéconomique (milieu favorisé $35 \%$, classe moyenne $33 \%$, milieu défavorisé $35 \%$ ) et ethnique (homogène $59 \%$, multi-ethnique $41 \%$ ).

L'analyse des représentations sociales visant la mise à jour des principes organisateurs communs à un groupe, les analyses se font traditionnellement par la classification descendante hiérarchique $(\mathrm{CDH})$ et l'analyse factorielle des correspondances $(\mathrm{AFC}$ ), qui reposent très souvent sur des épreuves d'association de mots (Doise, et al., 1992). La CDH et l'AFC ont été réalisées à l'aide du logiciel ALCESTE (Analyse des Lexèmes Cooccurrents dans un Ensemble de Segments de Textes) ${ }^{1}$.

1. La CDH permet, en plus de quantifier les structures signifiantes les plus fortes, d'associer des variables sociodémographiques ou illustratives à des classes de discours. Ces classes sont calculées en fonction d'un $\mathrm{Khi}^{2}$ d'association dans lequel intervient le nombre d'unités de contexte élémentaire (u.c.e.), lesquelles représentent la plus petite unité statistique définissable qui peut correspondre à un mot, une expression ou un segment de texte. Dans le cas d'épreuves d'associations de mots, une u.c.e. correspond donc à un mot. Le Khi ${ }^{2}$ d'association est calculé en fonction du nombre d'u.c.e. (mots) de la classe considérée, le nombre d'u.c.e. (mots) où le mot est présent et le nombre d'u.c.e. (mots) de la classe où le mot est présent (Reinert, 2003). L'analyse factorielle des correspondances (AFC) ajoute un niveau d'analyse supplémentaire. En effet, l'AFC, est une technique de décomposition du Khi ${ }^{2}$ en facteurs linéaires et la valeur propre ou l'inertie extraite par le facteur permet d'apprécier les valeurs les plus impliquées dans la mesure du $\mathrm{Khi}^{2}$. Le concept d'inertie est fondamental dans l'analyse factorielle des correspondances, car le nuage de points formé par les données que l'on veut décrire ne s'étend pas uniformément dans toutes les directions en raison des relations qui s'établissent entre les lignes et les colonnes regroupant les données. Un système de repère orthogonal plus avantageux, qui s'appuie sur la géométrie vectorielle, est utilisé pour repérer dans un nuage de points (coordonnées sur plusieurs dimensions des données dans un espace vectoriel) la représentation la plus fidèle possible d'une structure de données à plusieurs dimensions. On en calculera le profil 
Ainsi, ces analyses permettent de décrire, à l'aide de quelques classes (CDH : regroupement des mots induits partageant une valeur élevée d'association) et quelques facteurs (AFC), un tableau de cooccurences qui produit une représentation spatiale schématique de l'articulation des classes de discours, des variables socio-démographiques et des éléments les plus significatifs du discours associés à ces variables. Dans le cas de l'AFC, les axes factoriels sont interprétés comme des variables tensives (Pommier, 2004), qui révèlent des oppositions entre les classes de discours, et des variables sociodémographiques, qui caractérisent chacune des polarités des axes factoriels. L'analyse considère les induits de tous les inducteurs regroupés (aliment, manger, santé, malade) sans référence à chacun d'entre eux et permet de déterminer les différents profils indépendamment d'un inducteur particulier, ce qui n'est pas possible dans une analyse distincte de chacun des inducteurs. Dans le cas où l'analyse révèle des profils hétérogènes, il est intéressant de visualiser les frontières et les zones communes de chacun des profils, afin de dresser un portrait plus réaliste de l'univers représentationnel.

\section{PRÉSENTATION DES RÉSULTATS}

La classification descendante hiérarchique $(\mathrm{CDH})$ présentée au graphique 1 a été réalisée à partir des 2182 mots produits par les élèves de maternelle et de première année. Cette analyse a produit cinq classes formées par les modalités des variables sociodémographiques ${ }^{2}$ et les mots ou induits significatifs, soit ceux dont la valeur du $\mathrm{Khi}^{2}\left(\mathrm{X}^{2}\right)$ d'association est égale ou supérieure à 3,93.

moyen, soit le centre de gravité du nuage, qui maximise l'inertie du nuage de points. Du centre de gravité du nuage, on décompose l'inertie (dispersion projetée) totale du nuage comme la somme de l'inertie parallèle et de l'inertie perpendiculaire. Le premier axe factoriel est la ligne pour laquelle l'inertie parallèle est maximale. Le second axe factoriel sera, parmi toutes les droites orthogonales, celle pour laquelle la dispersion projetée (inertie) du nuage comme complément orthogonal est maximale. De façon formelle, l'inertie est la somme pondérée de la distance du $\mathrm{Khi}^{2}$ entre chaque profil d'individus et le profil moyen. Ainsi, plus grande est l'inertie, plus grande sera l'association des lignes et colonnes de données.

2. Modalité d'une variable sociodémographique: une catégorie (ex. : filles) liée à une variable (ex. : sexe) Modalité d'une variable active : mots induits par un inducteur. 


\section{Graphique 1}

Proportion des classes de mots induits

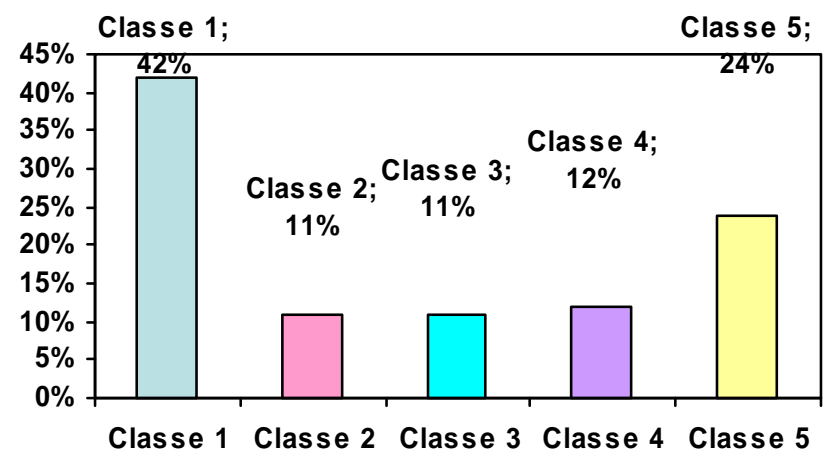

Comme on peut l'observer au graphique 1, les classes de mots induits varient de $11 \%$ à $42 \%$, la première et la dernière classe comportant le plus grand nombre de mots induits significatifs. Les contenus de chacune de ces classes sont présentés au prochain tableau.

Tableau 1

Description des contenus des classes

\begin{tabular}{|c|c|c|c|c|c|}
\hline $\begin{array}{l}\text { Classes } \\
\text { d'u.c.e. }\end{array}$ & $\begin{array}{l}\% \\
\text { des mots } \\
\text { induits }\end{array}$ & $\begin{array}{l}\text { Modalités des variables } \\
\text { sociodémographiques }\end{array}$ & $\mathrm{X}^{2}$ & $\begin{array}{l}\text { Modalités des } \\
\text { variables actives } \\
\text { (Mots induits) }\end{array}$ & $x^{2}$ \\
\hline Classe 1 & $42 \%$ & $\begin{array}{l}\text { Milieu défavorisé } \\
\text { Filles } \\
\text { 1ère année }\end{array}$ & $\begin{array}{l}149,24 \\
57,37 \\
41,82\end{array}$ & Manger & 46,47 \\
\hline Classe 2 & $11 \%$ & Garçons & 54,62 & $\begin{array}{l}\text { Hot dog } \\
\text { Médecin } \\
\text { Jouer } \\
\text { Repos } \\
\text { Hamburger } \\
\text { Mal de cœur }\end{array}$ & $\begin{array}{l}84,21 \\
54,62 \\
33,51 \\
33,51 \\
27,19 \\
6,49\end{array}$ \\
\hline Classe 3 & $11 \%$ & $\begin{array}{l}\text { Milieu favorisé } \\
\text { Garçons }\end{array}$ & $\begin{array}{l}26,10 \\
16,94\end{array}$ & $\begin{array}{l}\text { Mal de tête } \\
\text { Aliment } \\
\text { Frites } \\
\text { Carottes } \\
\text { Fruits }\end{array}$ & $\begin{array}{l}43,76 \\
38,22 \\
32,36 \\
27,69 \\
24,54\end{array}$ \\
\hline Classe 4 & $12 \%$ & $\begin{array}{l}\text { Garçons } \\
\text { Milieu multi-ethnique }\end{array}$ & $\begin{array}{l}30,28 \\
26,91\end{array}$ & $\begin{array}{l}\text { Mal de ventre } \\
\text { Fièvre }\end{array}$ & $\begin{array}{l}73,21 \\
29,58\end{array}$ \\
\hline Classe 5 & $24 \%$ & $\begin{array}{l}\text { Maternelle } \\
\text { Milieu favorisé }\end{array}$ & $\begin{array}{l}45,55 \\
45,11\end{array}$ & $\begin{array}{l}\text { Banane } \\
\text { Orange } \\
\text { Raisin }\end{array}$ & $\begin{array}{l}41,69 \\
29,43 \\
25,83\end{array}$ \\
\hline
\end{tabular}


Pour chacune des classes décrites au tableau 1, nous commenterons les modalités des variables sociodémographiques et les mots induits qui entretiennent les associations les plus étroites. Des cinq classes de discours décrites au tableau 1, c'est la première classe qui présente la plus étroite association entre des variables sociodémographiques et un mot particulier, en raison de la valeur du $\mathrm{Khi}^{2}\left(\mathrm{X}^{2}\right)$ la plus élevée. La première classe est axée sur la nourriture (manger), qui a été plus étroitement associée à l'ensemble de quatre mots inducteurs (aliment, manger, santé, malade) par les élèves de l'école se situant en milieu défavorisé, en raison de la valeur du $\mathrm{Khi}^{2}$ d'association qui est plus élevée $(149,24)$ que celles observées pour les filles $(57,37)$ et les élèves de première année $(41,82)$. Par ailleurs, on ne constate pas d'association à des mots liés à la santé ou à la maladie par les élèves fréquentant une école située dans un milieu défavorisé. La seconde classe apparaît plus diversifiée que les autres classes, car on y observe des associations significatives des mots ${ }^{3}$ qui touchent à la fois la nourriture (hot dog et hamburger), la santé et la maladie (médecin, mal de cœur) et l'activité (jouer, repos), mais elle n'est caractéristique que des garçons $\left(X^{2}: 54,62\right)$. La troisième classe, plus caractéristique des élèves fréquentant l'école située dans un milieu aisé $\left(X^{2}: 26,10\right)$ et moindrement des garçons $\left(X^{2}: 16,94\right)$, réfère d'abord à un symptôme (mal de tête) pour se concentrer ensuite sur la nourriture (aliment, frites, carottes, fruits). La quatrième classe, qui concerne encore les garçons et, cette fois, les élèves fréquentant une école située dans un milieu multi-ethnique, n'est caractérisée que par deux symptômes, dont le premier, le mal de ventre, est plus caractéristique de cette classe $\left(X^{2}: 73,21\right)$ que la fièvre $\left(X^{2}: 29,58\right)$. La cinquième et dernière classe se rapporte davantage aux fruits (banane, orange, raisin) et elle caractérise autant les élèves de maternelle $\left(X^{2}: 45,55\right)$ que ceux fréquentant une école située dans un milieu favorisé $\left(X^{2}: 45,11\right)$. L'analyse factorielle des correspondances (AFC) permet, comme on peut l'observer au graphique 2, d'établir la relation existant entre les cinq classes décrites précédemment. Chacune des modalités significatives est projetée sur le plan factoriel ainsi que sur le centre de gravité de la classe, qui est identifié par la lettre $\mathrm{C}$. On peut donc repérer l'opposition entre les classes sur les axes factoriels: la classe 5 s'opposant à la classe 1 sur le premier axe, et la classe 4 s'opposant aux classes 2 et 3 sur le deuxième axe. Par cette analyse, on peut également apprécier la dispersion des modalités d'une classe par rapport à son centre de gravité. Elle permet aussi d'estimer la proximité des modalités actives (les mots induits ou systèmes d'images) et des modalités sociodémographiques ${ }^{4}$, qui réfèrent à l'appartenance à un groupe. Cette proximité étant révélatrice d'une association plus étroite entre les mots ou systèmes d'images partagés par un ou des groupes donnés, l'AFC révèle donc les principes organisateurs communs des représentations sociales de l'alimentation, de la santé et de la maladie des jeunes enfants à l'étude.

3. Les valeurs du test $\mathrm{du} \mathrm{Khi}^{2}$ ne seront pas présentées systématiquement afin de ne pas alourdir le texte. Le lecteur peut se référer aux valeurs inscrites au tableau 1.

4. II est à noter que les valeurs du $\mathrm{Khi}^{2}$ sont inscrites au graphique 2 pour chacune des modalités significatives qui ont été projetées sur le plan factoriel. Toutefois, comme elles ont été décrites auparavant dans les cinq classes de discours, elles ne seront pas systématiquement reprises dans les commentaires sur l'analyse factorielle afin de ne pas alourdir inutilement le texte. 


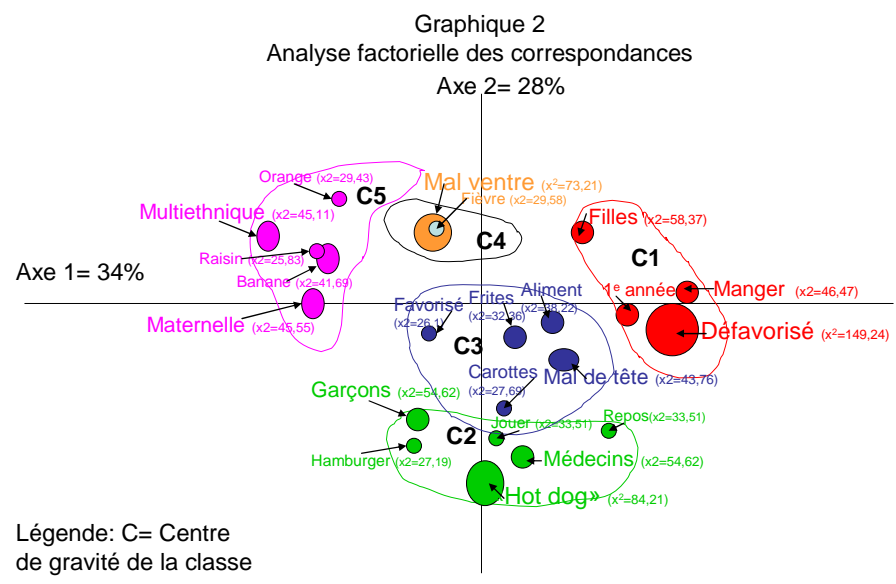

Les deux principaux facteurs d'organisation, dont l'articulation apparaît particulièrement complexe, sont responsables de $62 \%$ de l'inertie totale du nuage de points, ce qui révèle une assez forte association entre les profils de données. Le premier facteur (axe 1: $34 \%$ de l'inertie totale) demeure très centré sur l'alimentation, car il oppose la classe 5 axée sur les fruits et la classe 1 orientée sur l'action de manger. Cet axe correspond à une vision développementale de l'alimentation. Tout d'abord, on constate une étroite association entre le mot «manger » et les élèves fréquentant une école située en milieu défavorisé, ce qui révèle que ces élèves ont plus fréquemment que les autres associé le mot « manger » aux quatre mots inducteurs (aliment, manger, santé, malade). Ceci prévaut, mais dans une association moins étroite, pour les élèves de première année. Dans le cas des filles, cette modalité est aussi contributive au deuxième axe factoriel, ce qui lui donne une position située à mi-chemin entre le premier et second axe factoriel. Les filles ont donc associé les quatre mots inducteurs au mot «manger» mais aussi au mot «mal de ventre ». Si l'on se déplace à l'autre extrémité de l'axe, on constate que les élèves de maternelle et ceux de l'école multiethnique ne se situent pas à proximité l'un de l'autre. Ils partagent en commun une association plus étroite des mots inducteurs avec des fruits, particulièrement, la banane et le raisin. Par ailleurs, les élèves de l'école multi-ethnique ont choisi plus souvent l'orange que les élèves de la maternelle. Si on se rapporte à l'opposition des classes 5 et 1 sur l'axe 1, on constate l'effet d'une variable développementale, l'enfant se représentant d'abord l'alimentation en fonction de fruits (maternelle), symbolisés principalement par la banane, pour passer à une représentation de l'alimentation fondée sur l'acte de manger (1ère année). Ce dernier constat sera discuté en conclusion.

Tandis que le premier facteur révélait une première organisation des données en fonction de l'alimentation, le second facteur (axe 2: $28 \%$ de l'inertie totale) révèle des relations multiples se rapportant à la maladie, la santé et l'alimentation. Ce facteur correspond à une vision compartimentée de la maladie dans laquelle les éléments ne sont 
pas interreliés, mais juxtaposés. Ainsi le second axe factoriel oppose principalement la classe 4, liée au mal de ventre et à la fièvre, à deux autres classes, dont la contribution majeure sur la portion négative de l'axe 2 concerne la modalité «hot dog » de la classe 2. On constate donc dans un premier temps que cet aliment décrié en termes nutritionnels mais populaire chez les enfants, le hot dog, s'oppose le plus fortement à la maladie. Par ailleurs, les deux classes qui s'opposent à la classe 4 centrée sur la maladie ont des affinités statistiques, en raison de leur situation sur l'axe factoriel. Jouer est une modalité commune aux classes 2 et 3, et c'est donc par la santé que ces deux classes entretiennent des affinités. Par ailleurs, les garçons sont plus concernés par les éléments de la classe 2 (hot dog, médecin, jouer, repos), particulièrement le hamburger, qui est la modalité la plus voisine. Les élèves fréquentant une école située dans un quartier favorisé ont associé plus étroitement que les autres élèves, le mal de tête, les aliments pris dans un sens général, les carottes et les frites. II faut noter que ces élèves de milieu favorisé se situent à plus courte distance des frites, comme les garçons se situent à plus courte distance du hamburger. On retrouve donc dans les classes 2 et 3, des aliments déconseillés mais aimés des enfants, et des éléments liés à la santé. Ce qui diffère dans les deux classes, ce sont des modalités au statut plus ambigu de la classe 2 (repos, médecin) qui peuvent être liés à la maladie ou à la santé. On constate donc que les mets déconseillés aux jeunes (hot dog, hamburger et frites), parce qu'ils ne sont pas tous regroupés dans une même classe ou dans un même territoire factoriel, ne sont pas associés de la même façon par tous les enfants. De plus, le discours de mise en garde relatif à ces aliments ne semble pas intégré par les jeunes, car aucun de ces aliments n'avoisine directement une modalité telle que le mal de tête ou le mal de ventre. Bien au contraire, le mal de ventre s'oppose à tous ces aliments.

\section{DISCUSSION ET CONCLUSION}

Les principes organisateurs des représentations sociales de l'alimentation, de la santé et de la maladie des jeunes enfants à l'étude qui ont émergé de nos analyses (CDH et $\mathrm{AFC}$ ) sont constitués de deux axes, dont le premier correspond à une vision développementale de l'alimentation et le second à une vision compartimentée de la maladie. Par ailleurs, ces analyses ont également permis de faire émerger des éléments d'objectivation des représentations sociales de l'alimentation chez les jeunes enfants interrogés. Les représentations sociales étant par définition communes à un groupe, on constate que les éléments d'objectivation sont plus éclatés selon les groupes quand il s'agit de l'alimentation que de la maladie. Ainsi, la représentation sociale de l'alimentation des élèves de maternelle est objectivée par la banane, celle des élèves de l'école multi-ethnique par l'orange, celle des élèves de première année, des filles et des élèves de l'école se situant dans un milieu défavorisé par l'action de manger, celle des garçons par les hamburgers et hot dogs, et, enfin, celle des élèves de l'école située dans un milieu favorisé, plus étroitement par les frites que par les carottes. La représentation sociale de la maladie qui semble partagée par l'ensemble des jeunes enfants, est axée sur le mal de ventre et moindrement sur la fièvre, alors que les 
élèves de l'école située dans un milieu favorisé se représentent la maladie surtout par le mal de tête.

Conformément à la théorie des représentations sociales et aux résultats des études antérieures de Garnier et Doise (2002), on observe une différenciation des univers représentationnels selon le stade développemental, le milieu socio-économique et le sexe de l'enfant. Concernant le stade développemental, on repère nettement, sur le premier facteur, la distinction entre les élèves de maternelle plus attachés à l'énumération d'aliments, et ceux de première année plus axés sur l'action. Par ailleurs, la proximité des élèves de maternelle et des milieux ethniques pourrait s'expliquer par les difficultés des élèves du milieu ethnique à s'exprimer en français, ce qui les rapprocherait davantage des élèves de maternelle. L'énumération des catégories d'aliments leur serait plus aisée. D'autre part, la proximité des élèves de première année et ceux de l'école située en milieu défavorisé étonne au premier abord, les deux groupes étant fortement caractérisés par le mot « manger ». En outre, autant les élèves du milieu défavorisé que les élèves de première année sont soumis à des contraintes relatives à l'action de manger. Dans un cas, il s'agit de disponibilité de la nourriture, et dans l'autre cas, cette disponibilité est soumise à l'apprentissage des contraintes nouvelles quant aux périodes permises pour manger à l'école. Ainsi, le premier facteur ne concernerait pas uniquement le stade développemental, mais également des contextes d'apprentissage particuliers. De tels résultats confirment les résultats de Kubik (2003) sur l'influence des facteurs environnementaux intra et extrascolaires.

En ce qui a trait au milieu socio-économique, on aurait pu s'attendre, selon les préjugés véhiculés dans les milieux médicaux et scolaires, à une distinction des milieux favorisé/défavorisé, l'un étant axé sur les fruits et l'autre sur le repas rapide. Les résultats de l'analyse factorielle dissocie clairement les élèves du milieu favorisé (classe 3) de ceux du milieu multi-ethnique (classe 5), étroitement associé aux fruits. Les mets décriés par les autorités nutritionnelles, tels que le hamburger, le hot dog et les frites, ne sont pas non plus caractéristiques du stade de développement, car ils ne sont pas plus associés aux élèves de maternelle que ceux de première année. De plus, l'association de ces mets à la maladie n'est pas déterminante dans l'analyse, car il y intervient des éléments positifs, tels que le repos et le jeu. Cela pourrait confirmer l'hypothèse de la variabilité ou même de l'ambiguïté des messages destinés aux enfants. Cette ambiguïté des messages adressés aux enfants pourrait également expliquer l'absence de mots relatifs à l'obésité, aux allergies alimentaires et aux caries dentaires, qui font pourtant l'objet des campagnes de sensibilisation à l'école et dans divers médias. L'articulation équivoque des représentations sociales de l'alimentation, de la santé et de la maladie, en témoignant des interférences qui agissent dans l'environnement social des jeunes enfants, prêche en faveur des interventions en service social portant sur les aspects socioculturels de l'alimentation. 


\section{RÉFÉRENCES BIBLIOGRAPHIQUES}

ABRIC, J.C. (dir.) (1994). Pratiques sociales et représentations, Paris, PUF.

BIBER, D. (1996). «Investigating language use through corpus-based analyses of association patterns », International journal of corpus linguistics, vol. 1, $\mathrm{n}^{0} 2$, p. 171-197.

Bruss, M.B., J.R. MorRIS, L.L. Dannison, M.P. ORBE, J.A. QuituguA, et R.T. Palacios (2005). « Food, Culture, and Family: Exploring the Coordinated Management of Meaning Regarding Childhood Obesity », Health Communication, vol. 18, n 2, p. 155-175.

Bruss, M.B., J. MoRris, et L. DANNISON (2003). « Prevention of childhood obesity: sociocultural and familial factors », Journal of the American Dietetic Association, vol. 103, $\mathrm{n}^{\circ}$ 8, p. 10421045.

Cavallo, P.B., et A. Iannaccone (1993). «Représentations sociales et construction des connaissances », Papers on Social Representations, vol. 2, $n^{\circ}$ 3, p. 1-150.

Colver, A.F, H. Nevantaus, C.F. Macdougall, et A.J. Cant (2005). « Severe food-allergic reactions in children across the UK and Ireland, 1998-2000 », Acta Paediatric, vol. 94, n' 6, p. 689-695.

CORSARO, W.A. (1990). «The underlife of the nursery school: young childrens's social representations of adult rules », dans G. DUVEEN et B. LLOYD, Social representations and the development of knowledge, Cambridge, Cambridge University Press, p. 11-26.

Costalat-Founeau, A.M., M.C. Picot, D. Hauchard, M. Klimekova, et F. Favier (2002). «Représentation du corps et de l'alimentation chez une population de femmes de plus de 75 ans », Papers on Social Representations, vol. 11, $n^{\circ} 4,1-20$.

CouRTIAL, J.P. (1997). "La méthode des mots associés, outil d'analyse du changement social », Histoire et mesure, vol. 12, n ${ }^{\circ} 3-4$, p. 251-270.

DE RosA, A.S. (1988). «Sur l'usage des associations libres dans l'étude des représentations sociales de la maladie mentale », ERES, Tome 6, Connexions 51, p. 27-50.

Doise, W., A. Clémence, et F. Lorenzi-Cioldi (1992). Représentations sociales et analyses de données, Grenoble, Presses Universitaires de Grenoble.

DORTIER, J.F. (2003). «L'univers des représentations ou l'imaginaire de la grenouille », dans J.F. DORTIER (dir.), Le cerveau et la pensée: la révolution des sciences cognitives, Paris, Éditions Sciences humaines, PUF, p. 417-429.

DUVEEN, G. (1999). « Le développement des représentations sociales chez les jeunes enfants: un exemple, le genre », dans M.L. ROUQUETTE et C. GARNIER (dir.), La genèse des représentations sociales, Montréal, Éditions nouvelles, p. 114-135.

ELIADIS, E.E. (2006). «The Role of Social Work in the Childhood Obesity Epidemic », Social Work, vol. 51, $\mathrm{n}^{0} 1$, p. 86-88.

Ells, L.J., K. Campbell, J. Lidstone, S. Kelly, R. Lang, et C. Summerbell (2005). « Prevention of childhood obesity », Best Practice Resident Clinical Endocrinology Metabolism, vol. $19, n^{\circ} 3$, p. 441-454.

Garnier, C., N. Bednarz, et I. Ulanovskaya (dir.) (2004). Après Vygotski et Piaget. Perspectives sociale et constructiviste. Écoles russe et occidentale, Bruxelles, Éditions De Boeck, $2^{\mathrm{e}}$ édition 2004, 288 pages. 
GARNIER, C., et W. DoISE (dir.) (2002). Les représentations sociales : balisage du domaine d'études, Montréal, Éditions Nouvelles, 303 pages.

GARNIER, C. (2002). «La pensée sociale : questions vives », dans C. GARNIER (dir.), Les formes de la pensée sociale, Paris, Presses Universitaires de France, p. 1-17.

GARNIER, C., et M.L. RouQuetTE (dir.) (2000). Représentations sociales et éducation, Montréal, Éditions Nouvelles, 234 pages.

Holub, S.C., D.R. Musher-Eizenman, A.V. Persson, L.A. Edwards-Leeper, S.E. Goldstein, et A.B. MILLER (2005). « Do preschool children understand what it means to "diet," and do they do it? », International Journal of Eat Disorder, vol. 38, n 1 , p. 91-93.

IVIE, J.M. (2005). «The Beliefs of Case Managers about Elders' Use of Alcohol and Other Drugs: An Assessment of Theoretical Models of Substance Abuse », The Humanities and Social Sciences, vol. $65, n^{\circ} 10$, p. 2992-A.

Jacquinot, G. (dir.) (2002). Les jeunes et les médias. Perspectives de recherche dans le monde, Paris, L'Harmattan.

Jorm, A.F., H. Christensen, et K.M. Griffiths (2005). «Public beliefs about causes and risk factors for mental disorders: changes in Australia over 8 years », Psychiatry and Psychiatric Epidemiology, vol. 40, $\mathrm{n}^{\circ}$ 9, p. 764-767.

Koletzko B., J.P. GiRARdet, W. Klish, et O. Tabacco (2002). « Obesity in Children and Adolescents Worldwide: Current Views and Future Directions-Working Group Report of the First World Congress of Pediatric Gastroenterology, Hepatology, and Nutrition », Journal of Pediatric Gastroenterology et Nutrition, vol. 35, n 2, 205- 212.

KUBIK, M.Y. (2003). «Social environmental factors in the schools setting and their influence on young adolescent dietary behaviour », Dissertation Abstracts International, vol. 63, no 7B, p. 3258.

INSTITUT DE LA STATISTIQUE DU QuÉBEC (1999). Enquête sociale et de santé auprès des enfants et des adolescents québécois, Gouvernement du Québec, p. 311-344.

LAHLOU, S. (1994). Les représentations sociales de l'alimentation, Thèse de doctorat, École des Hautes Études en Sciences Sociales, Paris.

LehmanN, B.A. (2002). « Social Work Practitioners Comparative Evaluations of Obese and Lean Clients », The Humanities and Social Sciences, vol. 62, $n^{\circ} 12$, p. 4333-A.

LÉVY-Strauss, C. (1971). L'homme nu. Paris, Plon.

LoWES, J., et M. TIGgEMANN (2003). « Body dissatisfaction, dieting awareness and the impact of parental influence in young children », British Journal of Health Psychology, vol. 8, $\mathrm{n}^{\circ} 2$, p. 135-147.

Markova, I. (2000). « Amédée or How to Ger Rid of It: Social Representations from a Dialogical Perspective », Culture et Psychology, Sage Publications, vol. 6, n 4, p. 419-460.

Marshall, T.A., B. Broffitt, J. Eichenberger-Gilmore, J.J. Warren, M.A. CunNingham, et S.M. LEVY (2005). « The roles of meal, snack, and daily total food and beverage exposures on caries experience in young children », Public Health Dentist, vol. 65, n 3, p. 166-173.

MASSON, E., et S. Moscovici (1997). Les mutations dans la pratique alimentaire: processus symboliques et représentations sociales, Rapport de fin de recherche, Paris, Laboratoire de psychologie sociale. 
Mazza, C.S., B. OzunA, A.G. KrochiK, et M.B. Araujo (2005). « Prevalence of type 2 diabetes mellitus and impaired glucose tolerance in obese Argentinean children and adolescents », Journal of Pediatric Endocrinology Metabolism, vol. 18, $\mathrm{n}^{\circ}$ 5, p. 491-498.

Ministère de LA SANTÉ ET des SeRvices sociaux (MSSS) (2004). La nutrition et l'enfant, Gouvernement du Québec.

[http://www.msss.gouv.qc.ca/sujets/santepub /nutrition/index.html].

Morgan, J. (2005). « Nutrition for toddlers: the foundation for good health: Current problems and ways to overcome them », Journal of Familial Health Care, vol. 15, n 3, p. 85-88.

Moscovicl, S. (1993). «Introductory address », Papers on Social Representations, vol. 2, $\mathrm{n}^{\circ} 3$, p. $160-170$.

Moscovicı, S. (1990). «Social psychology and developmental psychology: extending the conversation », dans G. DUVEEN et B. LLOYD, Social representations and the development of knowledge, Cambridge, Cambridge University Press, p. 164-185.

POMmiER, J.L. (2004). Des variables tensives inscrites dans le texte : une interprétation dynamique de l'A.F.C. dans l'analyse d'Alceste, 7es Journées internationales d'analyse statistique des données textuelles, JADT.

PRovencher, Y. (2003). " Milieux sociaux d'appartenance et représentions de la santé chez un groupe de femmes âgées francophones du Nouveau-Brunswick », Revue Service social, vol. 50, no 1, p. 231-262.

RenNiE, K.L., L. Johnson, et A. JebB (2005). «Behavioural determinants of obesity », Best Practice Resident Clinical Endocrinology Metabolism, vol. 19, nº 3, p. 343-358.

REINERT, M. (2003). « Le rôle de la répétition dans la représentation du sens et son approche statistique par la méthode ALCESTE », Sémiotica, vol. 147, n 1/4, p. 389-420.

RouquetTe, M.L., et C. GARniER (dir.) (1999). La genèse des représentations sociales, Montréal, Éditions Nouvelles, 266 pages.

SAVoIE-ZAJC, L. (2005). «Children's Visual Representations of Food and Meal Time: towards an understanding of nutrition and educational practices », European Educational Research Journal, vol. 4, $n^{\circ} 2$, p. 132-141.

Sher, I., L. McGINN, J.A. SIREY, et B. MEYERS (2005). « Effects of Caregivers Perceived Stigma and Causal Beliefs on Patients Adherence to Antidepressant Treatment », Psychiatric Services, vol. 56, $n^{\circ} 5$, p. 564-569.

Sobal, J., L. Kettel-Khan, et C. Bisogni (1998). « A Conceptual Model of the Food and Nutrition System », Social Science Medecine, vol. 47, $\mathrm{n}^{0}$ 7, p. 853-863. 